\title{
Bruce Beutler wins the 2013 ASCI/Stanley J. Korsmeyer Award
}

ur understanding of the activation of the innate immune system, the body's first line of defense against infection, is based largely on the contributions of Bruce Beutler and his research group. Among his many accomplishments, Beutler isolated TNF, discovered its inflammatory properties, and cloned the LPS receptor. In 2011 he shared the Nobel Prize in Physiology or Medicine with Ralph Steinman and Jules Hoffmann. Beutler's work is further celebrated this year with the presentation of the 2013 ASCI/Stanley J. Korsmeyer Award. Beutler recently spoke to the JCI about his research career and scientific approach.

JCI: How did you decide to focus your research on the immune system?

Beutler: I was interested in nature from the time I was a small child, and by the age of 7, I found biology to be the most compelling subject of all. When I was in my early teens, my father allowed me to work in his laboratory, and I was excited to do real experiments addressing the function of biological systems. I began learning immunology in college, in the laboratory of Susumu Ohno, and in medical school, but it was quite by chance that I began to make my career in this area. That was the result of my work as a postdoctoral fellow: I purified a cytokine we then called "cachectin" and now know as TNF, and I determined that it mediated many of the inflammatory effects of LPS. This work led me to wonder about the nature of microbe sensing by the innate immune system, and to search for the LPS receptor.

JCI: Has finding the LPS receptor changed our understanding of how immunity works?

Beutler: We still hear the terms "danger," "irritant," and the like used in immunology and medicine. To a large extent, these words merely hide our ignorance of precise molecular facts. Complex immune phenomena (and I include inflammation among these) are traceable to definable events. LPS causes severe systemic inflammation, altering the expression of thousands of genes, but it does so in a highly specific manner, as a result of interaction with a receptor that evolved to detect it. A rather small number of related receptors collectively recognize other molecules, made by most of the infectious agents we might ever encounter. These receptors are the point of first informational contact between ourselves and the microbial world. They may also be responsible for sterile inflammation under some conditions, and one of the most important areas of immunology remains the search for endogenous ligands that activate Toll-like receptors. In short, the innate immune system has highly specific triggers and evolved to master self/non-self discrimination as effectively as the adaptive immune system did.

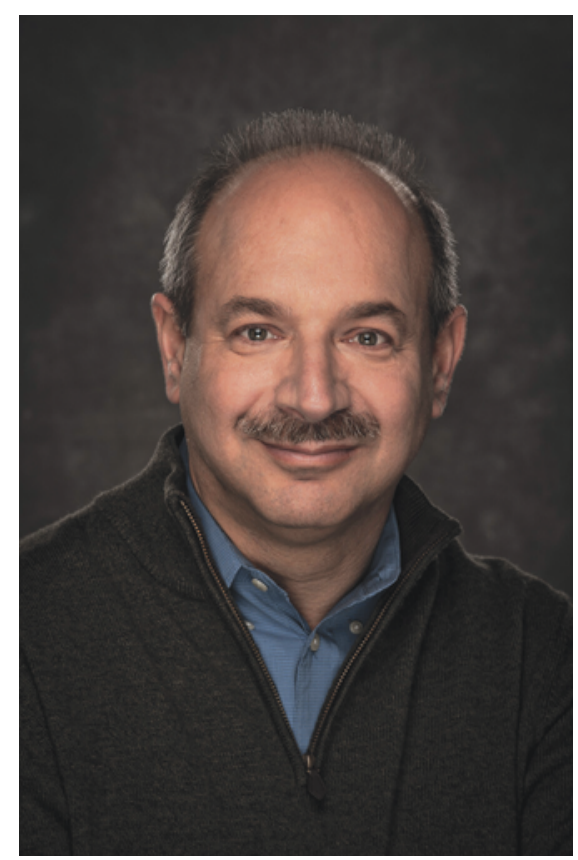

JCI: What part did you play in the development of etanercept?

Beutler: When I became convinced that TNF mediated endotoxin-induced shock, and by implication other forms of inflammation, I thought it would be useful to have a means of blocking it. I wished to enlist the TNF receptors, with their evolutionarily enforced specificity and lack of antigenicity, in the task of neutralizing TNF. A postdoctoral associate in my lab at UT Southwestern, Karsten Peppel, together with a graduate student, David Crawford, designed and expressed chimeric inhibitors in which the p55 or p75 TNF receptors were fused to an IgG heavy chain. These molecules worked beautifully, showing vastly higher avidity for TNF than an expressed receptor subunit alone, and circulating for weeks in mice. We patented them and sold the patent to Immunex, which later was acquired by Amgen. The product, etanercept, has helped many people with rheumatoid arthritis and other inflammatory diseases.

JCI: What motivated you to take a forward genetics approach to the study of immunity?

Beutler: When starting with phenotype there is no bias, and there is often great surprise as to what one finds. I adopted mutagenesis to create more phenotypic puzzles to solve, as the supply of "natural" phenovariance is limited (particularly on a defined genetic background). I felt we could explore almost any complex phenomenon in immunology simply by creating exceptions to it and finding the genetic cause. There's a certain joy in coming to work and learning about a new phenotype, perhaps never before seen by anyone else, and an equal joy in finding the cause. I started Mutagenetix to document and share these mutations, and we have contributed more than 100 of them to public repositories. Beyond that, Mutagenetix now lists many thousands of "incidental mutations" that alter specific proteins and may or may not cause a phenotype. We hope to collect mutations altering the function of every protein in the mouse.

JCI: How have technological advances changed that approach?

Beutler: Now one can sequence 40 or 50 mouse exomes per week in a single laboratory and thereby find the majority of influential mutations induced by ENU. No longer must we search in the dark for years to find the cause of phenotypes. We are trying to make it a "real-time" process, and we are very close to this goal. My hope is that our progress in deciphering immunity will be limited only by our imaginations and by the speed at which we can produce and screen mice. 
JCI: What was the greatest obstacle you've faced in your career?

Beutler: One thing we haven't yet discussed in this interview is the difficulty of the work for which the Nobel Prize was awarded. When we were searching for the Lps locus, the mouse genome was almost entirely terra incognita. There were very few crossovers in the relevant region of the chromosome: an unavoidable technical problem that prevented us from narrowing our critical region to less than $5 \mathrm{Mb}$ or so. It took us 5 years to discover the LPS recep- tor by positional cloning, and during that time many thought we were on a hopeless mission. Shortly before success came to us, my HHMI appointment was terminated, and a number of people drifted away from the lab. I began, bit by bit, to pay for the project out of my own pocket, because it was unthinkable that we should fail after all the hard work we had invested. It is fair to say that I was feeling extremely anxious until the day we found the mutation...and even for some time after that! Certainly my colleagues, Alexander Poltorak, Betsy Lay- ton, and Irina Smirnova, felt the same way. Those were wearing years, though at the same time extremely exciting.

JCI: What does receiving the Stanley J. Korsmeyer Award mean to you?

Beutler: I knew Stan Korsmeyer and greatly admired him. He might well have received a Nobel Prize had he not passed away at a young age. I am especially honored and moved to receive the prize that bears his name.

\section{Kathryn Claiborn}

